\title{
Barley varieties registered in the Czech Republic after the harvest of 2019
}

\author{
Vratislav Psota ${ }^{1 *}$, Olga Dvořáčková ${ }^{2}$, Markéta Musilová ${ }^{1}$, Milan Nečas $^{2}$ \\ Research Institute of Brewing and Malting, Mostecká 7 , \\ 61400 Brno, Czech Republic \\ 2 Central Institute for Supervising and Testing in Agriculture, \\ National Plant Variety Office, Hroznová 2, 65606 Brno, \\ Czech Republic \\ *corresponding author: psota@beerresearch.cz
}

\begin{abstract}
The study presents results of malting quality and agronomic characters determined within varieties of Avus, Fandaga, and LG Tosca after a three-year period of testing and Adam and LG Ester after four years of testing with the purpose of obtaining the registration of these spring barley varieties in the Czech Republic. Adam and LG Ester were recommended for the production of beer with the Protected Geographical Indication "České pivo". Extract from the varieties was at the levels of 82.6 and $81.9 \%$. They exhibited the required lower level of proteolytic modification and apparent final attenuation. Cytolytic modification was also low. Avus, Fandaga, and LG Tosca had a high extract in malt dry matter, which ranged from 83.4 to $84.1 \%$. The varieties had optimal to strong proteolytic modification (the Kolbach index of 46.8-55.6\%). Fandaga exhibited the highest content of free amino nitrogen (246 mg/l). Amylolytic and cytolytic modifications were at the optimal level. Wort quality was optimal (apparent final attenuation was between 82.1 and $83.2 \%$ ). LG Tosca always provided clear wort while other varieties provided weakling opalizing wort.
\end{abstract}

Keywords: barley, variety, malting quality

\section{Introduction}

New barley varieties are registered in the Czech Republic under Act 219/2003 on the basis of a three-year long period of testing which is carried out according to the Methodology of Barley Utility Value Testing (Dvořáčková, 2019). In the framework of the tests for the registration of new varieties, the utility value, i.e. yield and other yield characteristics, resistance to diseases, lodging and traits characterizing malt quality are monitored. Quality of malt samples in our study was assessed on the basis of the characters given in the Malting Quality Index (further only MQI) (Psota and Kosař, 2002).

In the last decades, malting barley varieties with strong enzymatic activity, high extract content and high degree of final apparent attenuation were preferred. For historical reasons, the Czech brewing industry has preserved a decoction production of pale lager. For the beer production of the Czech type, spring malting barley varieties are suit- able as they allow production of beer with a higher level of residual extract, strong palatefulness, excellent foaming and a relatively low alcohol content (Kosař et al., 2004).

Two groups of varieties are evaluated in this study: the varieties recommended for the production of beer with the Protected Geographical Indication (further only PGI) "České pivo"/Czech Beer (Adam and LG Ester) and the malting barley varieties with a high enzymatic activity (Avus, Fandaga, and LG Tosca).

\section{Material and Methods}

In the presented study technological and agronomic characters of spring barley varieties of Adam, Avus, Fandaga, LG Ester, and LG Tosca were assessed (Table 1). After the harvest of 2019 all these varieties were officially regis- 
Table 1 Spring barley varieties registered after the harvest of 2019

\begin{tabular}{|c|c|}
\hline Variety/Code & Maintainer/Agent in the $\mathrm{CR}$ \\
\hline spring barley & malting varieties \\
\hline Adam & NORDSAAT Saatzucht GmbH \\
\hline NORD 15/1107 & SAATEN - UNION CZ s.r.o. \\
\hline Avus & Saatzucht Streng - Engelen GmbH \& Co.KG \\
\hline STRG 687/15 & B O R, s.r.o. \\
\hline Fandaga & NORDSAAT Saatzucht GmbH \\
\hline NORD 14/2404 & SAATEN - UNION CZ s.r.o. \\
\hline LG Ester & Limagrain Europe \\
\hline LGBHE3254B & Limagrain Central Europe Cereals, s.r.o. \\
\hline LG Tosca & Limagrain Europe \\
\hline LGBN14223-2 & Limagrain Central Europe Cereals, s.r.o. \\
\hline winter barley & non malting varieties \\
\hline \multicolumn{2}{|r|}{ 6-row varieties } \\
\hline Beckenbauer & W. von Boriies-Eckendorf GmbH \& Co.KG \\
\hline BE2008024004D & Ing. Marian Špunar \\
\hline Camilla & Saatzucht Donau Ges. m.b.H.\& CoKG \\
\hline SZD 2213A & PROSEV s.r.o. \\
\hline KWS Wallace & KWS LOCHOW GMBH \\
\hline KW 6-1541 & SOUFFLET AGRO a.s. \\
\hline LG Zoro & Limagrain Europe \\
\hline LGBB15W003 & Limagrain Central Europe Cereals, s.r.o. \\
\hline Rumcajs & Saatzucht Streng-Engelen GmbH \& Co.KG \\
\hline STRG 568/15 & SELGEN, a.s. \\
\hline SU Lauvira & NORDSAAT Saatzucht GmbH \\
\hline NORD $13078 / 8$ & SAATEN - UNION CZ s.r.o. \\
\hline \multicolumn{2}{|r|}{ 2-row varieties } \\
\hline Neptun & Sejet Planteforaedling I/S \\
\hline SJ 128045 & SELGEN, a.s. \\
\hline Sobell & Sejet Planteforaedling I/S \\
\hline SJ 128113 & Limagrain Central Europe Cereals, s.r.o. \\
\hline
\end{tabular}

tered after a period of testing. Further, also non-malting two-row winter varieties of Neptun and Sobell and sixrow varieties of Beckenbauer, Camilla, KWS Wallace, LG Zoro, Rumcajs, and SU Lauvira were registered (Table 1). In non-malting varieties, the utility value is given only in a table without a verbal description (Table 5).

Malting quality of spring barley was assessed upon a micromalting test, following (bio)chemical analysis, and determination of technological parameters of malt and wort. The grain samples for the micromalting tests were delivered by the National Plant Variety Office of the Central Institute for Supervising and Testing in Agriculture (CISTA) in Brno between 2016 and 2019.

Information on agronomic characteristics of the malting and non-malting varieties was acquired within the state varietal tests of the Czech Republic from the testing stations belonging to CISTA and other collaborating institutions (Tables 4 and 5).

Samples of barley varieties ( $500 \mathrm{~g}$ ) were malted in the automatic micromalting equipment of KVM (Uničov, Czech Republic). The Research Institute of Brewing and Malting (further only RIBM), always uses the same regime of steeping, germination and kilning for varietal testing. A method traditionally used in the RIBM was employed for laboratory malting. The method is basically identical to the MEBAK method (2011), see Table 2.

Steeping was conducted in a steeping box. The temperature of both water and air was kept at $14.0^{\circ} \mathrm{C}$. The length of steeping was 5 hours on the first day and on 
Table 2 Conditions and schedule of malting

\begin{tabular}{|c|c|c|c|c|c|}
\hline & \multirow{2}{*}{ Time } & Temperature of & Temperature of & Fan & Air \\
\hline & & ingoing air & outgoing air & speed & recirculation \\
\hline & $\mathrm{h}$ & ${ }^{\circ} \mathrm{C}$ & ${ }^{\circ} \mathrm{C}$ & $\%$ & $\%$ \\
\hline Steeping & & & \multirow{7}{*}{14.0} & & \\
\hline Wet period & 5.0 & & & & \\
\hline Dry period & 19.0 & & & & \\
\hline Wet period & 4.0 & & & & \\
\hline Dry period & 20.0 & & & & \\
\hline Wet period & \multirow{2}{*}{ * 24.0} & & & & \\
\hline Dry period & & & & & \\
\hline Germination & 72.0 & & 14.0 & & \\
\hline \multirow{8}{*}{ Kilning } & 1.0 & 14.0 to 55.0 & 14.0 to 25.0 & \multirow{8}{*}{70} & 0 \\
\hline & 11.0 & 55.0 & 25.0 to 35.0 & & 0 \\
\hline & 1.0 & 55.0 to 60.0 & 40.0 to 45.0 & & 40 \\
\hline & 1.0 & 60.0 to 65.0 & 45.0 to 50.0 & & 40 \\
\hline & 2.0 & 65.0 to 70.0 & 50.0 to 55.0 & & 40 \\
\hline & 1.0 & 70.0 to 75.0 & 55.0 to 65.0 & & 40 \\
\hline & 1.0 & 75.0 to 80.0 & 65.0 to 78.0 & & 80 \\
\hline & 4.0 & 80.0 & 78.0 & & 80 \\
\hline
\end{tabular}

the second day it was 4 hours. On the third day the water content in germinating grains was adjusted to the value of $45 \%$ by steeping or spraying.

Germination was conducted in a germination box. The temperature during germination was $14.0^{\circ} \mathrm{C}$. The total time of steeping and germination was $144 \mathrm{~h}$.

Kilning was performed in a one-floor electrically heated kiln. The total kilning time was $22 \mathrm{~h}$, prekilning took place at $55{ }^{\circ} \mathrm{C}$, and the kilning temperature was maintained at $80^{\circ} \mathrm{C}$ for 4 hours.

The present study evaluates spring barley varieties registered in the Czech Republic after the harvest of 2019 according to the MQI (Psota and Kosař, 2002). In the case of the varieties recommended for the production of beer with the PGI "České pivo", the requirements for quality of malt and wort given in the application for the PGI "České pivo" were considered (Commission Regulation, 2008).

In the course of the above mentioned tests, we measured MQI parameters, i.e. nitrogenous substances in non-malted grain, extract in malt dry matter, relative extract at $45^{\circ} \mathrm{C}$, the Kolbach index, diastatic power, apparent final attenuation, friability, $\beta$-glucans in wort, wort clarity and haze. The tests were conducted according to the methods presented in MEBAK publications (2011) and by EBC Analysis Committee (2010). Wort clarity was determined visually and was assessed as follows: 1 = clear, 2 = weakly opalizing, 3 = opalizing (Table 3 ).

\section{Results}

Content of nitrogenous substances in barley non-malted grain is an important factor affecting malt quality. The adhesion between the starch granules and protein matrix reduces the rate of starch degradation during malting (Brennan et al., 1996; Zou et al., 2015). Holtekjølen et al. (2006) found a significant negative correlation between the content of nitrogenous substances and starch. The nitrogenous substance content in barley grain can be easily affected by the course of weather, farming practices, etc. For this reason, samples of the assessed varieties had an approximately similar content of nitrogenous substances preferable at the optimal level (10.2-11.0\%) or similar. Content of the nitrogenous substances in grain of the studied varieties ranged from $10.1 \%$ in LG Tosca to $11.4 \%$ in LG Ester.

Malt made from the barley grain with a higher content of nitrogenous substances provides a lower content of fermentable extract (Briggs, 1998). This relationship was partly observed in the studied varieties. Extract content in grain of the studied varieties ranged from $81.9 \%$ in LG Ester to $84.1 \%$ in LG Tosca (Table 3).

The Kolbach index informs about the successfulness of proteolysis and characterises a relationship between the total amount of nitrogenous substances in malt and the amount of nitrogenous substances that pass during mashing to wort. The Kolbach index 


\begin{tabular}{|c|c|c|c|c|c|c|c|c|c|c|c|c|c|c|c|c|c|}
\hline \multirow[t]{3}{*}{ Methods } & \multirow[t]{3}{*}{ Units } & \multirow[t]{3}{*}{ References } & $\stackrel{\circ}{\circ}$ & 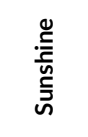 & 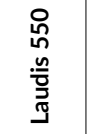 & 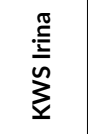 & 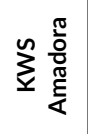 & $\frac{\varepsilon}{\frac{\pi}{0}}$ & 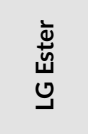 & $\stackrel{\text { ๕o }}{\circ}$ & 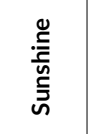 & 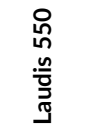 & $\frac{\stackrel{0}{\underline{\underline{S}}}}{\sum_{\underline{\Sigma}}^{n}}$ & 点 & 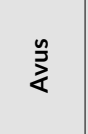 & $\begin{array}{l}\text { 品 } \\
\text { 胥 } \\
\text { एँ } \\
\text { एँ }\end{array}$ & 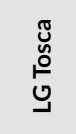 \\
\hline & & & \multicolumn{7}{|c|}{ 2016-2019 } & \multicolumn{8}{|c|}{ 2017-2019 } \\
\hline & & & $S$ & $S$ & $S$ & $S$ & $S$ & & & $S$ & $S$ & $S$ & $S$ & $S$ & & & \\
\hline Protein content of barley (factor 6.25) & $\%$ & EBC 2010 & 11.5 & 11.4 & 11.3 & 10.5 & 10.4 & 10.8 & 11.4 & 11.5 & 11.6 & 11.3 & 10.5 & 10.4 & 10.6 & 10.8 & 10.1 \\
\hline Starch content of barley & $\%$ & NIR & 63.7 & 63.0 & 63.5 & 63.8 & 63.9 & 63.3 & 63.3 & 63.6 & 62.7 & 63.4 & 63.6 & 63.7 & 63.4 & 63.7 & 63.9 \\
\hline Degree of steeping 1 & $\%$ & & 31.8 & 32.7 & 31.8 & 33.7 & 31.4 & 32.5 & 31.5 & 31.7 & 32.7 & 31.9 & 33.6 & 31.5 & 32.3 & 32.2 & 32.8 \\
\hline Degree of steeping 2 & $\%$ & & 39.3 & 40.4 & 39.3 & 41.6 & 39.2 & 40.0 & 39.2 & 39.4 & 40.3 & 39.4 & 41.5 & 39.3 & 40.0 & 40.1 & 40.8 \\
\hline Malt yield d. m. & $\%$ & Briggs 1998 & 91.2 & 90.9 & 91.2 & 90.6 & 91.2 & 91.8 & 91.7 & 91.1 & 90.8 & 91.2 & 90.4 & 91.0 & 91.5 & 91.4 & 90.5 \\
\hline Respiration losses d. m. & $\%$ & Briggs 1998 & 4.2 & 4.6 & 4.3 & 4.8 & 4.5 & 4.1 & 4.0 & 4.2 & 4.6 & 4.2 & 4.9 & 4.5 & 4.1 & 4.6 & 4.3 \\
\hline Rootlet losses d. m. & $\%$ & Briggs 1998 & 4.6 & 4.5 & 4.5 & 4.6 & 4.3 & 4.1 & 4.3 & 4.7 & 4.7 & 4.6 & 4.8 & 4.5 & 4.4 & 4.1 & 5.2 \\
\hline Extract of malt, congress mash & $\%$ & EBC 2010 & 82.2 & 82.2 & 82.1 & 82.4 & 83.6 & 82.6 & 81.9 & 82.3 & 82.1 & 82.2 & 82.5 & 83.5 & 83.4 & 83.4 & 84.1 \\
\hline Mash method according to Hartong and Kretschmer VZ $45^{\circ} \mathrm{C}$ & $\%$ & MEBAK 2011 & 37.4 & 43.0 & 38.4 & 42.6 & 50.6 & 41.8 & 40.5 & 37.4 & 43.5 & 38.6 & 43.0 & 51.0 & 44.3 & 52.0 & 48.4 \\
\hline Kolbach index & $\%$ & EBC 2010 & 41.3 & 48.7 & 42.8 & 46.6 & 52.3 & 41.4 & 42.3 & 40.7 & 48.3 & 42.5 & 46.5 & 52.2 & 46.8 & 55.6 & 48.9 \\
\hline Diastatic power & WK & EBC 2010 & 379 & 459 & 345 & 332 & 405 & 305 & 312 & 379 & 465 & 347 & 324 & 398 & 401 & 386 & 407 \\
\hline Final attenuation of laboratory wort from malt & $\%$ & EBC 2010 & 78.5 & 82.5 & 79.6 & 81.8 & 83.2 & 81.4 & 80.0 & 78.0 & 82.0 & 79.3 & 81.4 & 82.8 & 82.3 & 82.1 & 83.2 \\
\hline Friability & $\%$ & EBC 2010 & 83 & 91 & 83 & 86 & 98 & 81 & 77 & 81 & 88 & 81 & 84 & 97 & 95 & 96 & 91 \\
\hline High molecular weight $\beta$-glucan content of malt, FIA & $\mathrm{mg} / \mathrm{l}$ & EBC 2010 & 170 & 52 & 169 & 172 & 37 & 272 & 238 & 196 & 64 & 196 & 195 & 45 & 56 & 43 & 70 \\
\hline Protein content of malt (factor 6.25) & $\%$ & EBC 2010 & 10.7 & 10.6 & 10.4 & 9.6 & 9.4 & 9.9 & 10.5 & 10.8 & 10.9 & 10.6 & 9.7 & 9.6 & 10.0 & 10.0 & 9.3 \\
\hline Total nitrogen of malt, Kjeldahl method & $\%$ & EBC 2010 & 1.71 & 1.69 & 1.67 & 1.54 & 1.52 & 1.58 & 1.68 & 1.72 & 1.74 & 1.69 & 1.56 & 1.54 & 1.59 & 1.60 & 1.50 \\
\hline Soluble nitrogen of wort, Kjeldahl method & $\mathrm{mg} / \mathrm{l}$ & EBC 2010 & 785 & 940 & 801 & 805 & 894 & 729 & 783 & 780 & 940 & 801 & 805 & 894 & 835 & 997 & 817 \\
\hline Soluble nitrogen of wort, Kjeldahl method & $\mathrm{mg} / 100 \mathrm{~g}$ & EBC 2010 & 700 & 820 & 711 & 713 & 791 & 652 & 704 & 696 & 838 & 715 & 718 & 798 & 744 & 890 & 729 \\
\hline Soluble nitrogen of malt, Kjeldahl method & $\%$ & EBC 2010 & 0.700 & 0.838 & 0.715 & 0.718 & 0.798 & 0.652 & 0.701 & 0.696 & 0.838 & 0.715 & 0.718 & 0.798 & 0.744 & 0.890 & 0.729 \\
\hline Viscosity of laboratory wort from malt & mPa.s & EBC 2010 & 1.467 & 1.442 & 1.482 & 1.466 & 1.440 & 1.497 & 1.490 & 1.472 & 1.443 & 1.488 & 1.469 & 1.435 & 1.449 & 1.419 & 1.439 \\
\hline Colour of malt, visual method & EBC & EBC 2010 & 2.78 & 3.16 & 2.86 & 3.50 & 3.70 & 2.94 & 3.19 & 2.70 & 3.18 & 2.83 & 3.45 & 3.63 & 3.23 & 4.25 & 3.44 \\
\hline Saccharification time & $\min$ & EBC 2010 & 11 & 10 & 11 & 11 & 10 & 10 & 11 & 11 & 10 & 11 & 11 & 10 & 10 & 11 & 10 \\
\hline Glassy corns & $\%$ & EBC 2010 & 0.1 & 0.1 & 0.2 & 0.2 & 0.0 & 0.3 & 0.7 & 0.1 & 0.1 & 0.2 & 0.2 & 0.0 & 0.0 & 0.1 & 0.1 \\
\hline Partly unmodified grains & $\%$ & EBC 2010 & 1.8 & 0.5 & 1.7 & 1.2 & 0.1 & 3.8 & 4.5 & 2.1 & 0.6 & 2.2 & 1.3 & 0.1 & 0.2 & 0.2 & 0.5 \\
\hline Homogeneity (by friabilimeter) & $\%$ & Baxter, O'Farrell 1983 & 98.2 & 99.5 & 98.3 & 98.8 & 99.9 & 96.2 & 95.5 & 98.0 & 99.4 & 97.8 & 98.7 & 99.9 & 99.8 & 99.8 & 99.5 \\
\hline Appearance (clarity) of wort & & MEBAK 2011 & 1.06 & 1.06 & 1.06 & 1.00 & 1.06 & 1.25 & 1.06 & 1.08 & 1.08 & 1.08 & 1.00 & 1.00 & 1.08 & 1.17 & 1.00 \\
\hline Haze of wort $\left(90^{\circ}\right)$ & EBC & EBC 2010 & 0.91 & 0.85 & 0.98 & 0.89 & 1.13 & 1.42 & 1.12 & 1.01 & 0.84 & 1.04 & 0.81 & 0.82 & 0.94 & 1.44 & 0.68 \\
\hline Haze of wort $\left(12^{\circ}\right)$ & EBC & EBC 2010 & 0.94 & 1.04 & 1.09 & 1.00 & 1.14 & 1.52 & 1.42 & 1.01 & 1.04 & 1.13 & 0.93 & 0.96 & 1.22 & 1.47 & 0.72 \\
\hline Total polyphenols in wort & $\mathrm{mg} / \mathrm{l}$ & EBC 2010 & 66.4 & 70.0 & 64.3 & 88.8 & 89.0 & 68.0 & 75.6 & 66.4 & 67.8 & 64.1 & 86.4 & 87.8 & 71.9 & 75.0 & 75.6 \\
\hline Free amino nitrogen & $\mathrm{mg} / \mathrm{l}$ & EBC 2010 & 162 & 200 & 170 & 184 & 211 & 151 & 165 & 165 & 210 & 178 & 192 & 217 & 186 & 246 & 190 \\
\hline Free amino nitrogen & $\mathrm{mg} / 100 \mathrm{~g}$ & EBC 2010 & 148 & 187 & 159 & 172 & 194 & 140 & 153 & 148 & 187 & 159 & 172 & 194 & 165 & 219 & 170 \\
\hline
\end{tabular}


Table 4 Important agricultural properties of spring barley varieties

\begin{tabular}{|c|c|c|c|c|c|c|c|c|c|c|c|c|c|c|c|c|c|}
\hline \multirow[t]{2}{*}{ Variety } & \multirow[t]{2}{*}{ 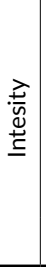 } & \multirow[t]{2}{*}{ 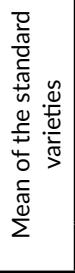 } & 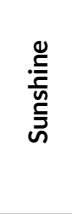 & 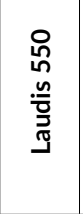 & 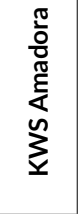 & 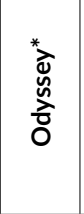 & 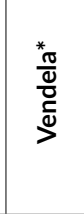 & $\frac{\varepsilon}{\frac{E}{\pi}}$ & $\begin{array}{l}\text { 离 } \\
\text { Uू } \\
\text { J }\end{array}$ & \multirow[t]{2}{*}{ 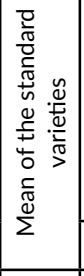 } & 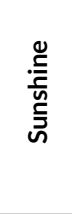 & 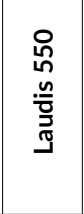 & 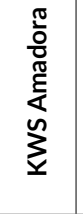 & 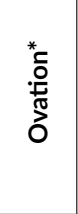 & $\stackrel{n}{\gtrless}$ & 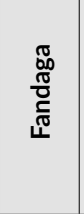 & 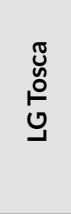 \\
\hline & & & \multicolumn{7}{|c|}{ 2016-2019 } & & \multicolumn{7}{|c|}{ 2017-2019 } \\
\hline \multicolumn{3}{|l|}{ Grain yield in (t/ha) } & S & $S$ & $S$ & $S$ & S & & & & S & $S$ & S & S & & & \\
\hline \multirow{2}{*}{$\begin{array}{l}\text { maize growing } \\
\text { region }\end{array}$} & $\mathrm{N}$ & 6.24 & 6.10 & 6.13 & 6.27 & 6.10 & 6.60 & 6.20 & 6.47 & 5.62 & 5.45 & 5.62 & 5.69 & 5.71 & 5.85 & 5.75 & 5.90 \\
\hline & $\mathrm{T}$ & 6.62 & 6.55 & 6.54 & 6.69 & 6.26 & 7.03 & 6.51 & 6.68 & 6.04 & 5.99 & 5.94 & 6.08 & 6.17 & 6.24 & 6.17 & 6.15 \\
\hline \multirow{2}{*}{$\begin{array}{l}\text { sugar beet and } \\
\text { cereal growing } \\
\text { regions }\end{array}$} & $\mathrm{N}$ & 7.36 & 7.23 & 7.32 & 7.32 & 7.39 & 7.56 & 7.61 & 7.56 & 7.14 & 6.98 & 7.07 & 7.15 & 7.35 & 7.49 & 7.32 & 7.51 \\
\hline & $\mathrm{T}$ & 7.75 & 7.62 & 7.54 & 7.76 & 7.80 & 8.02 & 7.85 & 7.76 & 7.52 & 7.43 & 7.32 & 7.54 & 7.80 & 7.79 & 7.67 & 8.11 \\
\hline \multirow{2}{*}{$\begin{array}{l}\text { potato and forage } \\
\text { growing regions }\end{array}$} & $\mathrm{N}$ & 6.71 & 6.29 & 6.55 & 6.97 & 6.97 & 6.78 & 6.79 & 6.90 & 6.75 & 6.39 & 6.61 & 6.97 & 7.03 & 7.17 & 6.97 & 7.24 \\
\hline & $\mathrm{T}$ & 7.54 & 7.22 & 7.20 & 7.92 & 7.45 & 7.91 & 7.75 & 7.44 & 7.68 & 7.53 & 7.28 & 7.99 & 7.92 & 8.31 & 8.24 & 8.24 \\
\hline \multicolumn{18}{|c|}{ Grain over $2.5 \mathrm{~mm}$ (t/ha) } \\
\hline \multirow{2}{*}{$\begin{array}{l}\text { maize growing } \\
\text { region }\end{array}$} & $\mathrm{N}$ & 4.96 & 4.88 & 5.05 & 4.62 & 5.06 & 5.18 & 5.25 & 5.45 & 4.14 & 4.22 & 4.49 & 3.80 & 4.06 & 4.83 & 4.11 & 4.35 \\
\hline & $\mathrm{T}$ & 5.33 & 5.53 & 5.20 & 5.19 & 5.16 & 5.56 & 5.43 & 5.50 & 4.38 & 4.59 & 4.41 & 4.09 & 4.43 & 5.11 & 4.27 & 4.56 \\
\hline \multirow{2}{*}{$\begin{array}{l}\text { sugar beet and } \\
\text { cereal growing } \\
\text { regions }\end{array}$} & $\mathrm{N}$ & 6.58 & 6.57 & 6.59 & 6.57 & 6.73 & 6.47 & 6.90 & 6.93 & 6.22 & 6.15 & 6.12 & 6.25 & 6.34 & 6.99 & 6.35 & 6.57 \\
\hline & $\mathrm{T}$ & 7.13 & 7.16 & 6.90 & 7.13 & 7.25 & 7.20 & 7.28 & 7.20 & 6.82 & 6.91 & 6.59 & 6.77 & 7.00 & 7.41 & 6.90 & 7.37 \\
\hline \multirow{2}{*}{$\begin{array}{l}\text { potato and forage } \\
\text { growing regions }\end{array}$} & $\mathrm{N}$ & 6.38 & 6.11 & 6.21 & 6.60 & 6.66 & 6.31 & 6.50 & 6.62 & 6.43 & 6.24 & 6.31 & 6.64 & 6.53 & 6.99 & 6.46 & 6.86 \\
\hline & $\mathrm{T}$ & 7.28 & 7.03 & 6.95 & 7.62 & 7.23 & 7.60 & 7.49 & 7.24 & 7.37 & 7.34 & 6.99 & 7.62 & 7.54 & 8.12 & 7.85 & 7.97 \\
\hline \multicolumn{18}{|c|}{ Agronomic data } \\
\hline \multicolumn{3}{|l|}{ straw length $(\mathrm{cm})$} & 74 & 73 & 70 & 72 & 73 & 74 & 73 & & 72 & 71 & 68 & 70 & 75 & 69 & 67 \\
\hline \multicolumn{3}{|l|}{ earliness of ripening** } & 113 & 112 & 112 & 113 & 112 & 112 & 112 & & 111 & 111 & 111 & 112 & 111 & 110 & 111 \\
\hline \multicolumn{3}{|l|}{$\begin{array}{l}\text { standing power } \\
\text { (lodging resistance) }\end{array}$} & 7.0 & 6.8 & 5.9 & 4.9 & 7.2 & 7.1 & 7.0 & & 7.3 & 7.3 & 6.7 & 6.7 & 7.6 & 7.3 & 7.5 \\
\hline \multicolumn{18}{|c|}{ Resistance to diseases } \\
\hline \multicolumn{3}{|l|}{$\begin{array}{l}\text { powdery mildew } \\
\text { (Blumeria graminis) }\end{array}$} & 5.8 & 8.9 & 8.9 & 8.9 & 6.6 & 8.8 & 8.8 & & 5.7 & 8.8 & 8.9 & 8.7 & 8.7 & 8.9 & 8.8 \\
\hline \multicolumn{3}{|c|}{$\begin{array}{l}\text { leaf brown rust of barley } \\
\text { (Puccinia hordei) }\end{array}$} & 7.1 & 6.3 & 5.0 & 6.1 & 7.2 & 6.8 & 6.9 & & 7.3 & 6.5 & 5.2 & 5.2 & 6.9 & 5.9 & 6.0 \\
\hline \multicolumn{3}{|c|}{$\begin{array}{l}\text { complex of leaf spot (Pyreno- } \\
\text { phora teres) }\end{array}$} & 6.9 & 6.0 & 6.7 & 6.6 & 5.5 & 6.5 & 6.3 & & 7.1 & 6.2 & 6.9 & 6.6 & 6.9 & 6.5 & 6.9 \\
\hline \multicolumn{3}{|c|}{$\begin{array}{l}\text { scald of barley } \\
\text { (Rhynchosporium secalis) }\end{array}$} & 8.0 & 7.3 & 7.1 & 8.1 & 6.0 & 8.3 & 7.7 & & 8.0 & 7.6 & 7.3 & 7.9 & 7.5 & 8.4 & 7.9 \\
\hline \multicolumn{3}{|c|}{$\begin{array}{l}\text { fusarium head blight (Fusarium } \\
\text { graminearum, F. culmorum, } \\
\text { Microdochium nivale etc.) }\end{array}$} & 5.4 & 7.0 & 6.5 & 6.8 & 5.9 & 5.4 & 7.1 & & 6.2 & 7.2 & 7.1 & 6.6 & 7.2 & 6.1 & 7.0 \\
\hline \multicolumn{3}{|c|}{$\begin{array}{l}\text { physiological leaf spots } \\
\text { of barley }\end{array}$} & 7.8 & 5.9 & 8.3 & 8.4 & 8.1 & 7.9 & 7.1 & & 7.6 & 6.0 & 8.3 & 8.1 & 7.9 & 8.5 & 8.5 \\
\hline & & & & & & & Grain & qualit) & & & & & & & & & \\
\hline 1000 grain weight (8 & & & 47 & 45 & 46 & 47 & 45 & 49 & 48 & & 47 & 44 & 45 & 46 & 51 & 46 & 45 \\
\hline $\begin{array}{l}\text { sieving fractions } \\
\text { over } 2.5 \mathrm{~mm}(\%) \\
\end{array}$ & & & 90 & 88 & 88 & 90 & 86 & 90 & 90 & & 88 & 86 & 85 & 84 & 92 & 85 & 86 \\
\hline $\begin{array}{l}\text { Comments: } \\
\text { Point evaluation } \\
1 \text { = fully lodging, full } \\
\text { Weight of } 1000 \text { grai } \\
{ }^{* *} \text { days from sowing } \\
\text { Intensity: } \\
\mathrm{N} \text { - non treated witl }\end{array}$ & & acked & m & orphore & egulat & 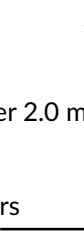 & $9=n$ & od & re & & ${ }^{*}$ Lin & eed da & 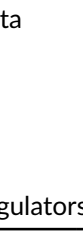 & & & & \\
\hline
\end{tabular}


Table 5 Important agricultural properties of winter barley varieties

\begin{tabular}{|c|c|c|c|c|c|c|c|c|c|c|c|c|c|c|c|}
\hline Variety & 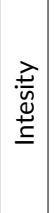 & 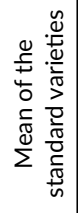 & 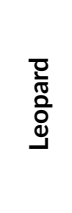 & $\begin{array}{l}\frac{\pi}{3} \\
\frac{0}{\pi} \\
\frac{0}{0}\end{array}$ & 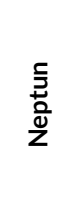 & $\begin{array}{l}\overline{\bar{\varpi}} \\
\text { 잉 }\end{array}$ & 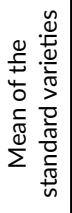 & 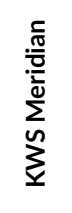 & $\stackrel{\varrho}{\stackrel{n}{=}}$ & 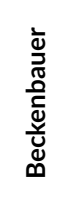 & $\begin{array}{l}\stackrel{\widetilde{\underline{E}}}{\overline{\bar{\varepsilon}}} \\
\stackrel{\bar{U}}{\tilde{U}}\end{array}$ & 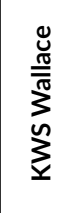 & $\begin{array}{l}\circ \\
\text { ㅇ } \\
\text { U }\end{array}$ & 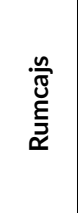 & 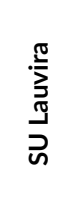 \\
\hline \multirow{2}{*}{ Number of rows } & \multicolumn{6}{|c|}{2} & \multicolumn{9}{|c|}{6} \\
\hline & & & $S$ & $S$ & & & & S & $S$ & & & & & & \\
\hline \multirow{2}{*}{ Grain yield (t/ha) } & $\mathrm{N}$ & 7.60 & 7.40 & 7.81 & 7.76 & 7.90 & 7.55 & 7.62 & 7.49 & 8.05 & 7.85 & 7.82 & 7.81 & 7.93 & 7.56 \\
\hline & $\mathbf{T}$ & 8.70 & 8.62 & 8.78 & 8.77 & 8.77 & 8.74 & 8.83 & 8.65 & 9.28 & 9.02 & 9.19 & 9.24 & 9.08 & 8.92 \\
\hline \multirow{2}{*}{ Grain over $2.5 \mathrm{~mm}$ (t/ha) } & $\mathrm{N}$ & 5.99 & 5.13 & 6.84 & 6.24 & 5.41 & 6.53 & 6.59 & 6.47 & 6.47 & 6.34 & 6.42 & 6.39 & 6.88 & 6.64 \\
\hline & $\mathbf{T}$ & 7.09 & 6.39 & 7.78 & 7.34 & 6.31 & 7.79 & 8.03 & 7.56 & 7.56 & 7.58 & 7.90 & 7.97 & 8.09 & 8.17 \\
\hline \multicolumn{16}{|c|}{ Agronomic data } \\
\hline \multicolumn{3}{|l|}{ earliness of ripening** } & 183 & 182 & 184 & 182 & & 182 & 183 & 182 & 181 & 183 & 182 & 182 & 182 \\
\hline \multicolumn{3}{|l|}{ number of ears $\left(\mathrm{pcs} / \mathrm{m}^{2}\right)$} & 894 & 873 & 950 & 993 & & 572 & 514 & 544 & 612 & 550 & 585 & 501 & 582 \\
\hline \multicolumn{3}{|l|}{ plant length $(\mathrm{cm})$} & 77 & 87 & 82 & 81 & & 95 & 103 & 94 & 88 & 92 & 97 & 97 & 93 \\
\hline \multicolumn{3}{|c|}{ standing power (lodging resistance) (9-1) } & 8.0 & 8.5 & 7.6 & 6.8 & & 7.4 & 8.0 & 8.2 & 8.4 & 7.7 & 6.5 & 8.1 & 7.9 \\
\hline \multicolumn{16}{|c|}{ Resistance to diseases (9-1) } \\
\hline \multicolumn{3}{|l|}{ powdery mildew (Blumeria graminis) } & 7.4 & 7.5 & 7.0 & 7.9 & & 7.1 & 7.8 & 6.8 & 6.1 & 6.5 & 7.6 & 7.3 & 8.1 \\
\hline \multicolumn{3}{|c|}{ leaf brown rust of barley (Puccinia hordei) } & 7.6 & 7.5 & 7.9 & 7.5 & & 7.3 & 7.5 & 7.0 & 6.9 & 6.5 & 6.2 & 6.5 & 7.4 \\
\hline \multicolumn{3}{|c|}{ complex of leaf spot (Pyrenophora teres) } & 6.4 & 7.1 & 6.6 & 7.5 & & 7.0 & 6.9 & 6.2 & 6.8 & 6.6 & 7.2 & 7.2 & 6.1 \\
\hline \multicolumn{3}{|l|}{ scald of barley (Rhynchosporium secalis) } & 6.1 & 8.1 & 7.8 & 8.3 & & 7.4 & 6.5 & 7.3 & 7.6 & 7.0 & 7.0 & 6.9 & 7.0 \\
\hline \multicolumn{3}{|c|}{$\begin{array}{l}\text { fusarium head blight (Fusarium graminearum, } \\
\text { F. culmorum, Microdochium nivale etc.) }\end{array}$} & 7.6 & 8.3 & 8.0 & 7.9 & & 7.4 & 7.4 & 7.4 & 7.2 & 7.3 & 6.7 & 7.5 & 7.1 \\
\hline \multicolumn{3}{|l|}{ physiological leaf spots of barley } & 6.9 & 7.3 & 6.8 & 7.8 & & 6.7 & 7.9 & 6.6 & 7.5 & 7.2 & 7.6 & 7.2 & 7.2 \\
\hline \multicolumn{16}{|c|}{ Grain quality } \\
\hline \multicolumn{3}{|l|}{ sieving fractions over $2.5 \mathrm{~mm}$ (\%) } & 68 & 86 & 79 & 67 & & 87 & 85 & 78 & 81 & 82 & 82 & 86 & 88 \\
\hline \multicolumn{3}{|l|}{1000 grain weight $(\mathrm{g})$} & 49 & 51 & 51 & 47 & & 45 & 48 & 43 & 44 & 46 & 46 & 46 & 46 \\
\hline \multicolumn{3}{|l|}{ bulk density (g/l) } & 642 & 658 & 662 & 645 & & 655 & 679 & 637 & 656 & 666 & 658 & 644 & 648 \\
\hline \multicolumn{3}{|l|}{ malting quality index $(9-1)$} & - & - & - & - & & - & - & - & - & - & - & - & - \\
\hline \multicolumn{16}{|c|}{$\begin{array}{l}\text { Comments: } \\
2 \text { - } 2 \text {-row } \\
S=\text { standard varieties } \\
\text { Point evaluation } \\
1 \text { = fully lodging, fully attacked } \quad 9 \text {-row } \\
\text { Weight of } 1000 \text { grains relates to sieving fractions over } 2.0 \mathrm{~mm} \text { at } 14 \% \text { humidity. } \\
{ }^{* *} \text { from } 1 . \text { January to maturity } \\
\text { Intensity: } \\
\mathrm{N} \text { - non treated with fungicides and morphoregulators }\end{array}$} \\
\hline
\end{tabular}

is one of the parameters that distinguish between the varieties recommended for the production of beer with the PGI "České pivo" from other malting varieties and this was also confirmed in this study. The values of The Kolbach index recorded in Avus, LG Tosca, and Fandaga were 5 to $14 \%$ higher than those detected in Adam and LG Ester (Table 3). Avus, LG Tosca, and Fandaga had a higher content of soluble nitrogenous substances in wort, with the highest content of soluble nitrogen (997 mg/l) recorded in Fandaga. As for the studied set of varieties, Fandaga also contained the highest content of free amino nitrogen in wort.
Relative extract at $45{ }^{\circ} \mathrm{C}$ is an indirect indicator of the activity of cytolytic and proteolytic enzymes. It represents the proportion of extract obtained at $45^{\circ} \mathrm{C}$, which is the optimal temperature for the activity of cytolytic enzymes. Also, in this parameter the varieties recommended for the production of beer with the PGI "České pivo" show lower values than Avus, Fandaga, and LG Tosca.

The activity of amylolytic enzymes hydrolysing starch, mainly $\beta$-amylase, was at the optimum level in the studied set of varieties. The varieties recommended for the production of beer with the PGI "České pivo" had 
a value of diastatic power several tens of WK un. lower than Avus, Fandaga, and LG Tosca.

The level of final apparent attenuation indicates the actual utilisation of the extract (Bathgate, 2016) and is affected by many factors (Koljonen et al., 1995). Apparent final attenuation is another parameter that clearly distinguishes the varieties recommended for the production of beer with the PGI "České pivo" from those with a high enzyme activity. Adam and LG Ester had the apparent final attenuation below $82 \%$, which is the value required for this group of varieties.

Cytolytic modification is an important factor affecting wort quality. The level of cell wall degradation is described by the following parameters: $\beta$-glucan content in wort, wort viscosity and malt friability. Worse levels of cytolytic modification affect negatively the value of apparent final attenuation (Edney et al., 1998). Lower friability values and a higher portion of glassy and semiglassy grains are indicators of weaker degradation of the endosperm (Allison et al., 1979). The average values of friability, glassy and semi-glassy grains indicate that Adam and LG Ester had a slower degradation of cell walls than Avus, Fandaga, and LG Tosca. The quantity of $\beta$-glucans in barley grain but also in wort has a huge impact on the technological quality of the relevant variety (Gupta et al., 2010). Avus, LG Tosca, and Fandaga had a fast degradation of cell walls and thus favourable values of the friability and $\beta$-glucan content. In the application for the PGI “České pivo" (Comission Regulation, 2008), cytolytic modification is assessed only by friability, which cannot be lower than 75\%. In case of LG Ester and Adam, low values of friability (77 and $81 \%$ ) correspond to a higher content of $\beta$-glucan in wort ( 238 and $272 \mathrm{mg} / \mathrm{l}$ ).

The parameters characterizing wort sensorial properties are wort haze measured with a nephelometer or wort clarity determined in a subjective way. Most malt houses operating in the territory of the Czech Republic require wort haze to be assessed for those varieties used in the registration period. In the studied varieties, the average value of wort haze at $90^{\circ} \mathrm{C}$ was measured around 1 EBC un., i.e. deeply below the limit of 4 EBC un. (Wackerbauer and Zufall, 1997). Only the variety of LG Tosca had clear wort in all cases. The other varieties gave weakly opalizing wort in some cases.

Adam bred in Germany provided malt with an above average content of extract $(82.6 \%)$ at the optimal nitrogenous substances content $(10.8 \%)$ in a non-malted grain. Proteolytic modification was above average (the Kolbach index of $41.4 \%$ ). Wort exhibited a content of soluble nitrogen at the level of $729 \mathrm{mg} / \mathrm{l}$. Free amino nitrogen content was at a lower level $(151 \mathrm{mg} / \mathrm{l})$, forming $21 \%$ of soluble nitrogen. Amylolytic modification was at the optimal level (diastatic power $305 \mathrm{WK}$ un.). Cytolytic modification was low. Degradation of cell walls was at the level of $81 \%$ and $\beta$-glucan content in wort moved around $272 \mathrm{mg} / \mathrm{l}$. The composition of wort was above average (apparent final attenuation of 81.4\%). In all cases the variety gave clear wort. The colour of wort corresponded to pale malt (EBC un.).

Adam has a malting quality with the point evaluation of 6 (5.6). The Research Institute of Brewing and Malting recommends the variety of Adam for the production of beer with the PGI "České pivo" as it fulfils the requirements given in the application for the PGI "České pivo" (Commission Regulation, 2008).

Adam is a mid early spring barley malting variety of mid high type, medium resistant to lodging, medium resistant to stem breaking. The grain size is medium big to big and portion of sieving fractions over $2.5 \mathrm{~mm}$ (90\%) is medium high to high. The variety is resistant to powdery mildew on the leaf, medium resistant to a complex of leaf brown rust of barley, medium resistant to a complex of leaf spots, resistant to scald, less resistant to fusarium head blight. There is a very high yield of sieving fractions over $2.5 \mathrm{~mm}$ in the non-treated variant when it is grown in sugar-beet and cereal areas, high in the non-treated variant grown in a maize area, medium high to high in the treated variant grown in sugar-beet and cereal areas, medium high in the treated variant grown in a maize area, and medium high in both variants grown in a potato area.

The utility value is given by a combination of a very high yield of sieving fractions which are over $2.5 \mathrm{~mm}$ in the non-treated variant when grown in sugar-beet and cereal areas, but high yield of sieving fractions over $2.5 \mathrm{~mm}$ in the non-treated variant grown in a maize area. Its malting quality meets the requirements for the production of beer with the PGI of "České pivo". Consequently, a comparison with other registered varieties shows that Adam variety has many benefits.

Malt from the variety of Avus bred in Germany provided a rich content of extract (83.4\%) at the optimal nitrogenous substance content $(10.6 \%)$ in a non-malted grain. Proteolytic modification was optimal (the Kolbach index of $46.8 \%$ ). Wort exhibited a high content of soluble nitrogen at the level of $835 \mathrm{mg} / \mathrm{l}$. Free amino nitrogen content was at a medium level $(186 \mathrm{mg} / \mathrm{l})$, forming $22 \%$ of soluble nitrogen. Amylolytic modification was at the optimal level (diastatic power $401 \mathrm{WK}$ un.). Cytolytic modification was optimal. Degradation of cell walls was at the level of $95 \%$ and $\beta$-glucan content in wort moved around $56 \mathrm{mg} / \mathrm{l}$. The composition of wort was optimal (apparent final attenuation of $82.3 \%$ ). In most cases the variety gave clear wort. The colour of wort corresponded to pale malt (3 EBC un.). 
Considering the values achieved in the studied technological parameters, Avus has a very good malting quality with the point evaluation of 9 (9.0).

According to the EU Plant variety database (European Commission 2019), the variety is registered in Austria and in 2019 it accomplished the state varietal tests in the Slovak Republic with similar results as in the Czech Republic (Psota et al., 2020).

Avus is a mid early to early spring barley malting variety, plants are mid high to high type, medium resistant to resistant to lodging, medium resistant to resistant to stem breaking. Its grain is big and portion of sieving fractions over $2.5 \mathrm{~mm}(92 \%)$ is high. The variety is resistant to powdery mildew on the leaf, medium resistant to a complex of leaf brown rust of barley, medium resistant to resistant to a complex of leaf spots, medium resistant to resistant to scald, medium resistant to fusarium head blight. There is a high yield of sieving fractions over $2.5 \mathrm{~mm}$ in both variants when they are grown in maize, sugar-beet and cereal areas and very high in the treated variant grown in a potato area, and between high to very high in the non-treated variant grown in a potato area.

The utility value is given by a combination of very high yield of sieving fractions over $2.5 \mathrm{~mm}$ in both variants grown in maize, sugar-beet and cereal areas and in the treated variant in a potato area, high to very high yield of sieving fractions over $2.5 \mathrm{~mm}$ in the non-treated variant grown in a potato area and very good malting quality. In comparison with the already registered varieties of spring barley, this one is apparently beneficial.

Fandaga bred in Germany provided malt with a rich content of extract $(83.4 \%)$ at the favourable nitrogenous substances content $(10.8 \%)$ in a non-malted grain. Proteolytic modification was strong (the Kolbach index $55.6 \%$ ). Wort exhibited a very high content of soluble nitrogen at the level of $997 \mathrm{mg} / \mathrm{l}$. Free amino nitrogen content was at a high level (246 mg/l), forming $25 \%$ of soluble nitrogen. Amylolytic modification was at the optimal level (diastatic power 386WK un.). Cytolytic modification was optimal. Degradation of cell walls was at the level of $96 \%$ and $\beta$-glucan content in wort moved around $43 \mathrm{mg} / \mathrm{l}$. The composition of wort was suitable (apparent final attenuation of $82.1 \%$ ). In most cases the variety gave clear wort. The colour of wort corresponded to pale malt (4 EBC un.).

Considering the values achieved in the studied technological parameters, Fandaga achieved a very good malting quality with the point evaluation of 5 (5.2).

According to the EU Plant Variety Database, the variety is registered in Estonia, Finland, France, Lithuania, and Poland.
Fandaga is a mid early malting variety of mid high to low type, medium resistant to lodging, medium resistant to stem breaking. Its grain is medium big and portion of sieving fractions over $2.5 \mathrm{~mm}$ is medium high. The variety is resistant to powdery mildew on the leaf, medium resistant to a complex of leaf brown rust of barley, medium resistant to a complex of leaf spots, resistant to scald, medium resistant to resistant to fusarium head blight. There is a high yield of sieving fractions over $2.5 \mathrm{~mm}$ in the treated variant when grown in a potato area, medium high in both variants grown in sugar-beet and cereal areas and in the non-treated variant in a potato area, between medium high and low in the non-treated variant when grown in a maize area, and low in the treated variant grown in a maize area.

The utility value is given by a combination of a high yield of sieving fractions over $2.5 \mathrm{~mm}$ in the treated variant grown in a potato area, malting quality and high content of free amino nitrogen. In comparison with the registered varieties of spring barley, this variety has apparent benefits.

LG Ester bred in the Czech Republic provided malt with a below average content of extract (81.9\%) at the mildly increased content of nitrogenous substances content $(11.4 \%)$ in a non-malted grain. Proteolytic modification was optimal (the Kolbach index of $42.3 \%$ ). Wort exhibited soluble nitrogen at the level of $783 \mathrm{mg} / \mathrm{l}$. Free amino nitrogen content was at a medium level ( $165 \mathrm{mg} / \mathrm{l})$ forming $21 \%$ of soluble nitrogen. Amylolytic modification was at the optimal level (diastatic power $312 \mathrm{WK}$ un.). Cytolytic modification was low. Degradation of cell walls was at the level of $77 \%$ and $\beta$-glucan content in wort moved around $238 \mathrm{mg} / \mathrm{l}$. The composition of wort was below average (apparent final attenuation of $80.0 \%$ ). In most cases the variety gave clear wort. The colour of wort corresponded to pale malt (3 $\mathrm{EBC}$ un.).

LG Ester has malting quality with the point evaluation of 4 (4.1). The Research Institute of Brewing and Malting recommends the variety LG Ester for the production of beer with the PGI "České pivo" as it fulfils the requirements given in the application for PGI "České pivo" (Commission Regulation, 2008).

LG Ester is a mid early spring barley variety of mid high type, medium resistant to lodging, medium resistant to resistant to stem breaking. Its grain is medium big, portion of sieving fractions over $2.5 \mathrm{~mm}$ is medium high to high. The variety is resistant to powdery mildew on the leaf, medium resistant to a complex of leaf brown rust of barley, medium resistant to a complex of leaf spots, medium resistant to resistant to scald, medium resistant to fusarium head blight. There is a very high yield of sieving fractions over $2.5 \mathrm{~mm}$ in the non-treated variant 
grown in maize, sugar-beet and cereal areas, high in the non-treated variant grown in a potato area, between medium high and high in the treated variant of grown in a maize area, and medium high in the treated variant grown in sugar-beet, cereal and potato areas.

The utility value is given by a combination of a very high yield of sieving fractions over $2.5 \mathrm{~mm}$ in the non-treated variant growing in maize, sugar-beet and cereal areas, high yield of sieving fractions over $2.5 \mathrm{~mm}$ in the non-treated variant grown in a potato area. Its malting quality meets the requirements for the production of beer with the PGI of "České pivo". In comparison with the registered varieties of spring barley, this one has apparent benefits.

LG Tosca bred in Holland provided malt with a rich content of extract $(84.1 \%)$ at a slightly lower content of nitrogenous substances (10.1\%) in a non-malted grain. Proteolytic modification was slightly increased (The Kolbach index of $48.9 \%$ ). Wort exhibited high content of soluble nitrogen at the level of $817 \mathrm{mg} / \mathrm{l}$. Free amino nitrogen content was at a higher level $(190 \mathrm{mg} / \mathrm{l})$, forming $23 \%$ of soluble nitrogen. Amylolytic modification was at the optimal level (diastatic power $407 \mathrm{WK}$ un.). Cytolytic modification was optimal. Degradation of cell walls was at the level of 91\% and $\beta$-glucan content in wort moved around $70 \mathrm{mg} / \mathrm{l}$. The composition of wort was suitable (apparent final attenuation of $83.2 \%$ ). The variety always gave clear wort. The colour of wort corresponded to pale malt (3 EBC un.).

Considering the values achieved in the studied technological parameters, Tosca achieved a very good malting quality with the point evaluation of 8 (8.5).

LG Tosca is a mid early malting variety. Plants are low, the variety is medium resistant to resistant to lodging, medium resistant to stem breaking. Its grain is big to small and portion of sieving fractions over $2.5 \mathrm{~mm}$ (86\%) is medium high. The variety is resistant to powdery mildew on the leaf, medium resistant to a complex of leaf brown rust of barley, medium resistant to a complex of leaf spots, resistant to scald, medium resistant to fusarium head blight. There is a very high yield of sieving fractions over $2.5 \mathrm{~mm}$ in the treated variant grown in sugar-beet and cereal areas, between high and very high in the treated variant grown in a potato area, high in the non-treated variant grown in sugar-beet, cereal and potato areas, and medium high in both variants grown in a maize area.

The utility value is given by a combination of a very high yield of sieving fractions over $2.5 \mathrm{~mm}$ in the treated variant grown in sugar-beet and cereal areas, high to very high yield of sieving fractions over $2.5 \mathrm{~mm}$ in the treated variant grown in a potato area, high yield of sieving fractions over $2.5 \mathrm{~mm}$ in the non-treated variant grown in sugar-beet, cereal and potato areas. Thanks to a very good malting quality, it meets the requirements for the production of beer with the PGI of "České pivo" and therefore, in comparison with the registered spring barley varieties, the variety has apparent benefits.

\section{Conclusion}

The study presents results achieved by five varieties which were registered in the Czech Republic after the harvest of 2019. Their quality was assessed according to the Malting Quality Index. Content of nitrogenous substances in the studied varieties of spring barley was at the optimal to mildly increased level (10.1 to $11.4 \%$ ). The spring barley varieties of Adam and LG Ester recommended for the production of beer with the PGI "České pivo" had extract contents, apparent final attenuation and cytolytic modification lower than the other tested varieties. Extracts higher than $83 \%$ were recorded in the varieties of Avus, Fandaga, and LG Tosca. LG Tosca showed the average extract content at the level of $84.1 \%$. Proteolytic, modification in the studied varieties was favourable, only Fandaga exhibited strong proteolytic modification and the highest content of free amino nitrogen. Diastatic power in all the studied varieties was at the optimal level. Apparent final attenuation and cytolytic modification in Avus, Fandaga, and LG Tosca were at the optimal level.

\section{Acknowledgement}

The submitted study was funded by the institutional support for a long-term strategic development of the research organization provided by the Ministry of Agriculture of the CR (R01918) and was supported by members of the Czech Beer and Malt Association and variety owners.

\section{References}

Act No. 219/2003 Coll., on the marketing of seed and planting material of cultivated plants and on the amendment to certain acts.

Allison, M. J., Borzucki, R., Cowe, I. A., McHale, R., 1979. Variation in a Barley Collection for Endosperm Attributes that Relate to Malting Quality. J. Inst. Brew. 85(2), 86-88. https://doi. org/10.1002/j.2050-0416.1979.tb06831.x

Baxter, E. D., O'Farrell, D. D., 1983. Use of the friabilimeter to assess homogeneity of malt. J. Inst. Brew. 89(3), 210-214. https://doi. org/10.1002/j.2050-0416.1983.tb04169.x

Bathgate, G. N., 2016. A review of malting and malt processing for whisky distillation: Malting and malt processing for whisky distillation. J. Inst. Brew. 122(2), 197-211. https://doi.org/10.1002/jib.332

Brennan, C., Harris, N., Smith, D., Shewry, P., 1996. Structural differences in the mature endosperms of good and poor malting barley cultivars. J. Cereal Sci. 24(2), 171-177. https://doi.org/10.1006/jcrs.1996.0050 
Briggs, D. E., 1998. Malts and Malting. Springer Science \& Business Media. ISBN: 0412298007

Commission Regulation, 2008. Publication of an application pursuant to Article 6(2) of Council Regulation (EC) No 510/2006 on the protection of geographical indications and designations of origin for agricultural products and foodstuffs. Official Journal of the European Union C 16-22

Dvořáčková, O., 2019. Metodika zkoušek užitné hodnoty. Ječmen. Brno, ÚKZÚZ NOÚ. http://eagri.cz/public/web/file/112373/Priloha_10_ ZUH10_2019_Jecmen.pdf

Edney, M. J., LaBerge, D. E., Langrell, D. E., 1998. Relationships Among the $\beta$-glucan Contents of Barley, Malt, Malt Congress Extract, and Beer. J. Am. Soc. Brew. Chem. 56(4), 164-168. https://doi.org/10.1094/ ASBCJ-56-0164

EBC Analysis committee, 2010. Analytica EBC, Barley: 3.2 Moisture Conten of Barley, 3.3.2 Total Nitrogen Content of Barley, Malt: 4.2 Moisture Content of Malt, 4.3.2 Total Nitrogen of Malt, 4.5.1 Extract of Malt, 4.9.1 Soluble Nitrogen of Malt, 4.12 Diastatic Power of Malt, 4.15 Friability, 4.16.2 High Molecular Weight Beta-glucan Content of Malt and Malt Wort, Beer: 9.29 Haze in Beer: Calibration of Haze Meters. Nüremberg: Fachverlag Hans Carl, 2010, 794 p. ISBN 978-3418-00759-5.

European Commission, 2019. Common catalogue of varieties of agricultural plant species -37 th complete edition. Official Journal of the European Union C 13, 11.1.2019.

Gupta, M., Abu Ghannam, N., Gallaghar, E., 2010. Barley for brewing: Characteristic changes during malting, brewing and applications of its by-products. Compr. Rev. Food Sci. Food Saf. 9(3), 318-328. https:// doi.org/10.1111/j.1541-4337.2010.00112.x

Holtekjølen, A. K., Uhlen, A. K., Bråthen, E., Sahlstrøm, S., Knutsen, S. H., 2006. Contents of starch and non-starch polysaccharides in barley varieties of different origin. Food Chem. 94(3), 348-358. https://doi. org/10.1016/j.foodchem.2004.11.022
Kosař, K., Psota, V., Mikyška, A., 2004. Barley Varieties Suitable for Production of the Czech-type Beer. Czech J. Genet. Plant Breed. 40(4), 137-139. https://doi.org/10.17221/3712-CJGPB

Koljonen, T., Hämäläinen, J. J., Sjöholm, K., Pietilä, K., 1995. A model for the prediction of fermentable sugar concentrations during mashing. J. Food Eng. 26(3), 329-350. https://doi.org/10.1016/02608774(94)00061-D

MEBAK, 2011. Raw material. 1 Barley: 1.5.3 Micromalting; Malz: 3.1.4.11 Maischmethode nach Hartong-Kretschmer VZ $45^{\circ} \mathrm{C}$. Mitteleuropäischen Brautechnischen Analysenkommission, Freising-Weihenstephan, Germany.

Plant variety database https://ec.europa.eu/food/plant/plant_propagation_material/plant_variety_catalogues_databases/search/public/ index.cfm?event=SearchForm\&ctl_type=A

Psota, V., Kosař, K., 2002. Malting Quality Index. Kvasny prumysl 48(6), 142-148. https://doi.org/0.18832/kp2002011

Psota, V., Svorad, M., Musilová, M., Halásová, V., 2020. Barley varieties registered in the Slovak Republic after harvest 2019. Kvasny prumysl 66(2), 255-260. https://doi.org/10.18832/kp2019.66.255

Wackerbauer, K., Zufall, C., 1997. From Malthouse to Brewery - Malt Specification and Analysis. Brauwelt International 15(1), 22-29.

Zou, W., Sissons, M., Gidley, M J., Gilbert, R. G., Warren, F. J., 2015. Combined techniques for characterising pasta structure reveal how the gluten network slows enzymic digestion rate, Food Chem. 188(1), 559-568. https://doi.org/10.1016/j.foodchem.2015.05.032 